
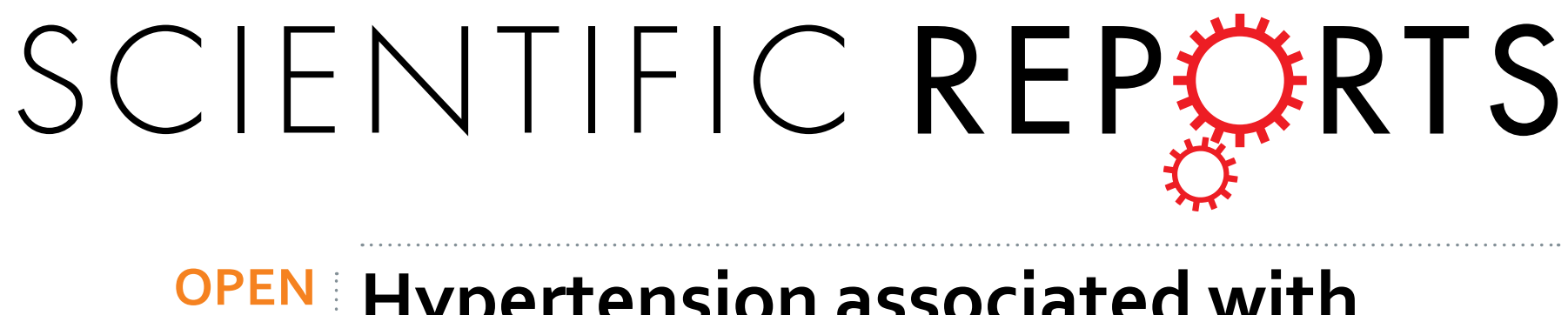

\title{
Hypertension associated with venous thromboembolism in patients with newly diagnosed \\ lung cancer
}

Received: 16 October 2015

Accepted: 14 December 2015

Published: 22 January 2016
Yuhui Zhang ${ }^{1}$, Yuanhua Yang ${ }^{1}$, Wenhui Chen ${ }^{1}$, Lirong Liang ${ }^{1}$, Zhenguo Zhai ${ }^{1}$, Lijuan Guo ${ }^{1}$, Chen Wang ${ }^{1,2}$ \& China Venous Thromboembolism (VTE) Study Group ${ }^{\#}$

The aim of this study was to evaluate associations between cardiovascular disease (CVD) risk factors and the occurrence of venous thromboembolism (VTE) in patients with lung cancer that might help estimate an individual's risk for VTE. A total of 632 unselected patients with newly diagnosed lung cancer were investigated for VTE within the three months prior to recruitment, and their major CVD risk factors were assessed at the baseline examination. Eighty-six of the 632 (13.6\%) developed a VTE event. Multivariate logistic regression analysis, including age, sex, smoking, body mass index, diabetes, dyslipidemia, hypertension and white blood cell count, found that hypertension (OR 1.8; 95\% $\mathrm{Cl} 1.0-3.3$ ) and leukocytosis (OR 2.7; 95\% Cl 1.5-4.8) were significantly associated with VTE in different tumor histology models and that hypertension (OR 1.9; $95 \% \mathrm{Cl} 1.1-3.4$ ) and leukocytosis (OR 2.7; 95\% Cl 1.5-4.7) were also significantly associated with VTE in different tumor stage models. Leukocytosis was linearly associated with hypertension and VTE ( $P$ for trend $=0.006$ ), and the ORs for VTE increased with leukocytosis (all $P$ for trend $<0.05$ ). In conclusion, hypertension increased the risk of VTE in patients with newly diagnosed lung cancer, which may be mediated by the presence of inflammation.

Venous thromboembolism (VTE) is one of the highest complications in patients with solid tumor, especially in lung cancer ${ }^{1-3}$. Classic risk factors for cancer-associated VTE include aging, poor performance status, prior history of VTE, histology of cancer, origin of cancer, chemotherapy, surgery, and other treatment-related factors $^{1,4-7}$. Although the risk factors potentially responsible for this disorder are identifiable in most patients, cancer-associated VTE remains unexplained in some patients.

Cardiovascular disease (CVD) risk factors, such as smoking, obesity, hypertension, dyslipidemia, and diabetes, often coexist with cancer. The risk of arterial thrombosis in patients with major CVD risk factors is most likely mediated by the presence of an inflammatory state and hypercoagulability ${ }^{8}$. Both increased inflammation and coagulation may predispose these patients to develop VTE events ${ }^{9}$. Furthermore, metabolic syndrome increases the risk of developing multiple types of cancer and $\mathrm{VTE}^{10-13}$. We hypothesized that cancer patients with CVD risk factors may be at increased risk of VTE. To test this hypothesis, we evaluated associations between major CVD risk factors and occurrence of VTE in patients with newly diagnosed lung cancer.

\section{Results}

Patient characteristics. A total of 691 unselected patients with newly diagnosed lung cancer were enrolled in this study. Sixteen patients were excluded because they had a history of deep vein thrombosis (DVT) or pulmonary embolism (PE) more than three months before recruitment. Another forty-three patients were excluded because they were continuously taking anticoagulants. In the end, 632 eligible patients were included in our study (Fig. 1).

\footnotetext{
${ }^{1}$ Beijing Institute of Respiratory Medicine, Beijing Key Laboratory of Respiratory and Pulmonary Circulation Disorders, Beijing Chao-Yang Hospital, Capital Medical University, Beijing 100020, China. ${ }^{2}$ National Clinical Research Center of Respiratory Medicine, China-Japan Friendship Hospital, Beijing 100029, China. "A comprehensive list of authors and affiliations appear at the end of the paper. Correspondence and requests for materials should be addressed to C.W. (email: zhangyhcy86@hotmail.com)
} 


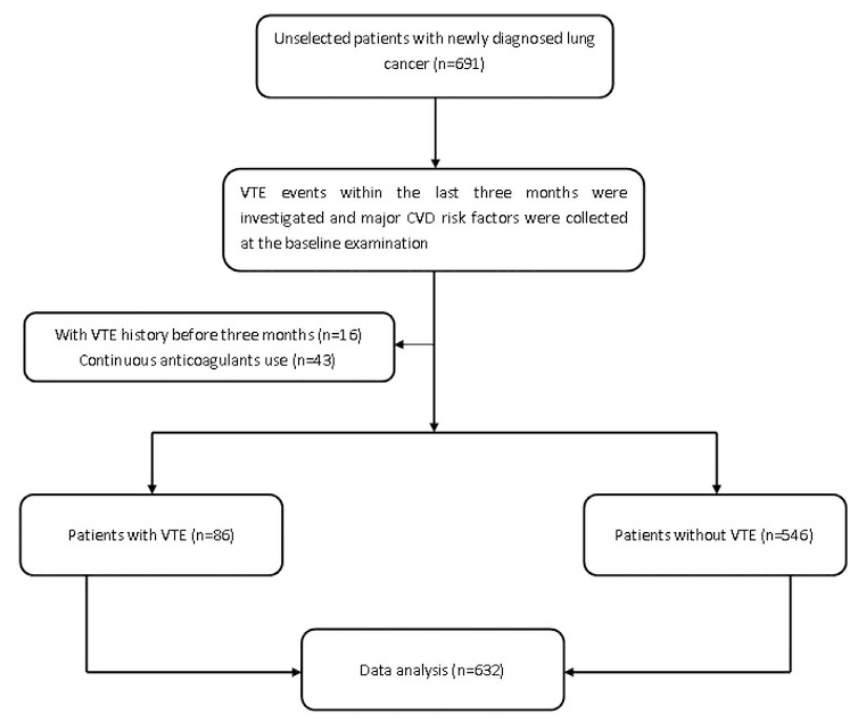

Figure 1. Study flow diagram. Abbreviations: VTE = venous thromboembolism; $C V D=$ cardiovascular disease.

The 632 included lung cancer patients had a median age of 63.5 years, and $71.7 \%$ of the patients were male. The baseline demographic and clinical characteristics of the investigated study population are listed in Table 1 . To analyze non-small cell lung cancer and small cell lung cancer collectively, the tumors were histologically categorized as adenocarcinoma or non-adenocarcinoma (all lung cancers with the exception of adenocarcinoma), and the tumor stage was categorized as localized stage (confined to ipsilateral hemithorax) or distant metastasis. The study population consisted of patients with adenocarcinoma $(n=295)$ and patients with non-adenocarcinoma $(\mathrm{n}=337)$. Distant metastases were found in 276 patients $(43.7 \%)$.

VTE events. Overall, 86 of the 632 patients (13.6\%) experienced a VTE event. 40 patients (6.3\%) developed lower-extremity DVT alone, 32 patients (5.1\%) developed PE alone, and 14 patients (2.2\%) developed both DVT and PE.

CVD factors and risk of VTE. We evaluated the associations between major CVD risk factors (age, sex, smoking, body mass index, diabetes, hypertension, total cholesterol, triglycerides, and white blood cell count) and risk of VTE in our study. VTE occurred more frequently in patients with hypertension or leukocytosis than in patients without them $(P<0.05$ for both). Detailed information for the patients with and without VTE is provided in Table 2.

Subsequently, we performed a multivariate logistic regression analysis (model 1) that included the age, sex, smoking, body mass index, diabetes, hypertension, dyslipidemia, and white blood cell count of newly diagnosed lung cancer patients with different tumor histologies (adenocarcinoma vs. non-adenocarcinoma) to identify factors associated with VTE (Table 3). Hypertension (vs. without, odds ratio [OR] 1.8; 95\% CI 1.0-3.3; P=0.041) and leukocytosis (vs. WBC $<10 \times 10^{9} / \mathrm{L}, \mathrm{OR} 2.7 ; 95 \%$ CI $1.5-4.8 ; P=0.001$ ) were significantly associated with VTE. Furthermore, we performed a multivariate logistic regression analysis (model 2) that included the age, sex, smoking, body mass index, diabetes, hypertension, dyslipidemia, and white blood cell count of newly diagnosed lung cancer patients with different tumor stages (localized stage vs. distant metastasis) to identify factors associated with VTE (Table 4). Hypertension (vs. without, OR 1.9; 95\% CI 1.1-3.4; $P=0.029$ ) and leukocytosis (vs. WBC $<10 \times 10^{9} / \mathrm{L}$, OR $2.7 ; 95 \%$ CI $\left.1.5-4.7 ; P=0.001\right)$ were also significantly associated with VTE.

Finally, we analyzed the associations of leukocytosis and hypertension with VTE. The patients were classified as four subgroups (no hypertension and no VTE, only hypertension, only VTE, or both hypertension and VTE) according to the inflammation load. The rate of leukocytosis was significantly different in the four subgroups $(P=0.04)$ and linearly associated with hypertension and VTE $(P$ for trend $=0.006)$. The ORs for VTE risk increased among the four subgroups (all $P$ for trend $<0.05$; Table 5 ).

\section{Discussion}

In the study population of newly diagnosed lung cancer patients, hypertension was associated with risk of VTE, which may be mediated through inflammation. Age, sex, smoking, body mass index, diabetes, and dyslipidemia were not associated with VTE risk. The same results were found for patients with different tumor histologies and tumor stages.

Whether CVD risk factors increase VTE risk has been a focus of many cohort and case-control studies ${ }^{14-17}$. Obesity has been demonstrated to be an independent risk factor of a VTE event ${ }^{18-21}$, whereas conflicting results have been reported for smoking, hypertension, dyslipidemia, and diabetes ${ }^{8,20,21}$. However, we were not able to confirm an association between high body mass index and VTE. In our study, the 632 lung cancer patients included in this study had a lower mean body mass index of $23.3\left(\mathrm{~kg} / \mathrm{m}^{2}\right)$ than the patients included in a previous 


\begin{tabular}{|c|c|c|}
\hline Characteristic & $\begin{array}{c}\text { Number of } \\
\text { patients }\end{array}$ & $\%$ \\
\hline Median age, years & 63.5 & \\
\hline 25th-75th percentile & $56-71$ & \\
\hline$<60$ & 213 & 33.7 \\
\hline$\geq 60$ & 419 & 66.3 \\
\hline \multicolumn{3}{|l|}{ Sex } \\
\hline Female & 179 & 28.3 \\
\hline Male & 453 & 71.7 \\
\hline \multicolumn{3}{|l|}{ Smoking } \\
\hline Never & 277 & 43.8 \\
\hline Former & 96 & 15.2 \\
\hline Current & 259 & 41.0 \\
\hline \multicolumn{3}{|l|}{ Body mass index, $\mathrm{kg} / \mathrm{m}^{2}$} \\
\hline Mean $(S D)$ & $23.3(3.4)$ & \\
\hline Diabetes & 49 & 7.8 \\
\hline Hypertension & 105 & 16.6 \\
\hline \multicolumn{3}{|l|}{ Total cholesterol , mmol/L } \\
\hline Mean $(S D)$ & $4.4(1.1)$ & \\
\hline \multicolumn{3}{|l|}{ Triglycerides, mmol/L } \\
\hline Mean $(S D)$ & $1.3(0.7)$ & \\
\hline \multicolumn{3}{|l|}{ WBC count } \\
\hline$<10 \times 10^{9} / \mathrm{L}$ & 534 & 84.5 \\
\hline$\geq 10 \times 10^{9} / \mathrm{L}$ & 98 & 15.5 \\
\hline \multicolumn{3}{|l|}{ Tumor histology } \\
\hline Adenocarcinoma & 295 & 46.7 \\
\hline Non-Adenocarcinoma & 337 & 53.3 \\
\hline Squamous cell carcinoma & 179 & 28.3 \\
\hline Other NSCLC & 57 & 9.0 \\
\hline SCLC & 101 & 16.0 \\
\hline \multicolumn{3}{|l|}{ Tumor Stage } \\
\hline Localized & 332 & 52.5 \\
\hline Distant metastasis & 276 & 43.7 \\
\hline Unknown & 24 & 3.8 \\
\hline
\end{tabular}

Table 1. Baseline demographic and clinical characteristics of the total study population $(n=632)$.

Abbreviations: $\mathrm{SD}=$ standard deviation; NSCLC $=$ non-small cell lung cancer; $\mathrm{SCLC}=$ small cell lung cancer; $\mathrm{WBC}=$ white blood cell.

study ${ }^{19}$, and $43.7 \%$ of the patients had distant metastases. The risk of obesity may be weakened due to weight loss and malnutrition in advanced lung cancer patients with tumor progression and metastasis.

Most studies agree that current smoking is associated with increased VTE risk ${ }^{19,20,22-25}$. Biologically, the association of smoking with VTE may be mediated through hypercoagulability and impaired fibrinolysis ${ }^{22,26}$. A Danish study found a dose-response relationship between current smoking and VTE risk. Former smokers have the same risk of VTE as patients who have never smoked, indicating the acute effects of smoking and underscoring the potential benefits of smoking cessation ${ }^{23}$. However, our study failed to show an association between smoking with VTE, which is in agreement with the results of some previous studies ${ }^{18,27}$. Smoking contributes to the development of cancer. Regarding smoking history in our study, $15.2 \%$ of the patients were former smokers, $41 \%$ of the patients were current smokers, and $43.8 \%$ of patients were non-smokers. The risk of smoking may be underestimated because of the pooling of ex-smokers with current smokers, a lack of distinction between light and heavy smokers, and a shorter follow-up time in this study.

The roles of diabetes and dyslipidemia in the pathogenesis of VTE are controversial ${ }^{8,18,19,21,22,28-30}$. Our analyses showed no correlation between diabetes or dyslipidemia and risk of VTE. The findings of our study are in agreement with published reports ${ }^{8,19,21,22,29}$. In this study, $7.8 \%$ and $3.3 \%$ of the patients experienced diabetes and dyslipidemia, respectively, and these rates are less than the rates obtained in previous studies. This difference is also likely because our study population consists of advanced lung cancer patients whose tumor growth and metastasis may deteriorate their nutritional status.

Interestingly, our findings corroborate the documented finding that hypertension is associated with increased VTE risk. The ORs for hypertension were less robust than those reported for established major risk factors for cancer-associated VTE, such as tumor histology and tumor stage. However, their coexistence increased the risk of VTE additionally. This result supported the previous finding that hypertension was associated with increased risk of $\mathrm{VTE}^{15,22}$. Hypertension was a common comorbidity in patients with lung cancer because $16.6 \%$ of the patients 


\begin{tabular}{|c|c|c|c|}
\hline Variable & $\begin{array}{l}\text { No VTE } \\
(\mathrm{n}=546)\end{array}$ & $\begin{array}{c}\mathrm{VTE} \\
(\mathbf{n}=86)\end{array}$ & $p$ \\
\hline Age, year (\%) & & & 0.464 \\
\hline$<60$ & $181(33.2)$ & $32(37.2)$ & \\
\hline$\geq 60$ & $365(66.8)$ & $54(62.8)$ & \\
\hline Gender (\%) & & & 0.797 \\
\hline Female & $156(28.6)$ & $23(26.7)$ & \\
\hline Male & $390(71.4)$ & $63(73.3)$ & \\
\hline Smoking history (\%) & & & 0.452 \\
\hline Never & $242(44.3)$ & $35(40.7)$ & \\
\hline Former & $81(14.8)$ & $15(17.4)$ & \\
\hline Current & $223(40.9)$ & $36(41.9)$ & \\
\hline Body mass index,kg/m² & & & 0.439 \\
\hline Mean $(S D)$ & $23.2(3.5)$ & $23.6(3.1)$ & \\
\hline Diabetes (\%) & & & 0.285 \\
\hline Yes & $40(7.3)$ & $9(10.5)$ & \\
\hline No & $506(92.7)$ & $77(89.5)$ & \\
\hline Hypertension (\%) & & & 0.043 \\
\hline Yes & $84(15.4)$ & $21(24.4)$ & \\
\hline No & $462(84.6)$ & $65(75.6)$ & \\
\hline Total cholesterol, $\mathrm{mol} / \mathrm{L}$ & & & 0.802 \\
\hline Mean (SD) & $4.4(1.1)$ & $4.4(1.0)$ & \\
\hline Triglycerides, mol/L & & & 0.400 \\
\hline Mean $(S D)$ & $1.3(0.6)$ & $1.3(0.9)$ & \\
\hline WBC count (\%) & & & 0.001 \\
\hline$<10 \times 10^{9} / \mathrm{L}$ & $472(86.4)$ & $62(72.1)$ & \\
\hline$\geq 10 \times 10^{9} / \mathrm{L}$ & $74(13.6)$ & $24(27.9)$ & \\
\hline Tumor histology (\%) & & & 0.001 \\
\hline Adenocarcinoma & $240(44.0)$ & $55(64.0)$ & \\
\hline \multicolumn{4}{|l|}{ Non-adenocarcinoma } \\
\hline Squamous cell carcinoma & $161(29.5)$ & $18(20.9)$ & \\
\hline Other NSCLC & $51(9.3)$ & $6(7.0)$ & \\
\hline SCLC & $94(17.2)$ & $7(8.1)$ & \\
\hline Tumor Stage (\%) & & & 0.014 \\
\hline Localized stage & $296(54.2)$ & $36(42.7)$ & \\
\hline Distant Metastasis & $226(41.4)$ & $50(57.3)$ & \\
\hline Unknown & $24(4.4)$ & $0(0)$ & \\
\hline
\end{tabular}

Table 2. Comparison of demographic and clinical characteristics between patients with and without VTE. Abbreviations: VTE = venous thromboembolism; SD = standard deviation; NSCLC $=$ non-small cell lung cancer; $\mathrm{SCLC}=$ small cell lung cancer; $\mathrm{WBC}=$ white blood cell.

\begin{tabular}{|l|c|c|c|}
\hline Patients Group & OR & 95\%CI & $P$ \\
\hline Hypertension & & & 0.041 \\
\hline Yes & 1.8 & $1.0-3.3$ & \\
\hline No & 1.0 & & \\
\hline WBC count & & & 0.001 \\
\hline$\geq 10 \times 10^{9} / \mathrm{L}$ & 2.7 & $1.5-4.8$ & \\
\hline$<10 \times 10^{9} / \mathrm{L}$ & 1.0 & & \\
\hline Tumor histology & & & 0.001 \\
\hline Adenocarcinoma & 2.3 & $1.4-3.8$ & \\
\hline Non-adenocarcinoma & 1.0 & & \\
\hline
\end{tabular}

Table 3. Factors associated with increased VTE risk in the multivariate logistic regression model (model 1) among newly diagnosed lung cancer patients with different tumor histologies*. ${ }^{*}$ The variables were entered simultaneously into the multivariate logistic regression model and included age, gender, smoking, body mass index, diabetes, hypertension, dyslipidemia, WBC count, and tumor histology (adenocarcinoma vs. non-adenocarcinoma). Only variables with $P$ values less than 0.05 are shown in the table. Abbreviations: $\mathrm{VTE}=$ venous thromboembolism; $\mathrm{OR}=$ Odds Ratio; $\mathrm{WBC}=$ white blood cell. 


\begin{tabular}{|l|c|c|c|}
\hline Patients Group & OR & 95\%CI & $\boldsymbol{P}$ \\
\hline Hypertension & & & 0.029 \\
\hline Yes & 1.9 & $1.1-3.4$ & \\
\hline No & 1.0 & & \\
\hline WBC count & & & 0.001 \\
\hline$\geq 10 \times 10^{9} / \mathrm{L}$ & 2.7 & $1.5-4.7$ & \\
\hline$<10 \times 10^{9} / \mathrm{L}$ & 1.0 & & \\
\hline Tumor stage & & & 0.016 \\
\hline Distant metastasis & 1.8 & $1.1-2.9$ & \\
\hline Localized stage & 1.0 & & \\
\hline
\end{tabular}

Table 4. Factors associated with increased VTE risk in the multivariate logistic regression model (model 2) among newly diagnosed lung cancer patients with different tumor stages ${ }^{*}$. ${ }^{*}$ The variables were entered simultaneously into the multivariate logistic regression model and included age, gender, smoking, body mass index, diabetes, hypertension, dyslipidemia, WBC count, and tumor stage (localized stage vs. distant metastasis). Only variables with $P$ values less than 0.05 are shown in the table. Abbreviations: VTE $=$ venous thromboembolism; $\mathrm{OR}=$ Odds Ratio; $\mathrm{WBC}=$ white blood cell.

\begin{tabular}{|l|c|c|c|c|}
\hline \multirow{2}{*}{ Patients Group } & \multicolumn{2}{|c|}{ Leukocytosis $^{1}$} & \multicolumn{3}{|c|}{ OR(95\%CI) } \\
\cline { 2 - 5 } & Number of patients (\%) & Unadjusted & Adjusted $^{2}$ & Adjusted $^{3}$ \\
\hline No hypertension and no VTE & $63(13.6)$ & 1 & 1 & 1 \\
\hline Only hypertension & $11(13.1)$ & $0.95(0.48-1.90)$ & $0.87(0.42-1.79)$ & $0.90(0.43-1.88)$ \\
\hline Only VTE & $20(30.8)$ & $2.82(1.56-5.08)$ & $3.51(1.67-5.96)$ & $3.21(1.69-6.08)$ \\
\hline Both hypertension and VTE & $4(19.0)$ & $1.49(0.49-4.57)$ & $1.33(0.41-4.39)$ & $1.33(0.41-4.36)$ \\
\hline$P$ for trend & 0.006 & 0.006 & 0.011 & 0.009 \\
\hline
\end{tabular}

Table 5. Leukocytosis and ORs for VTE among newly diagnosed lung cancer patients. ${ }^{1}$ Leukocytosis was defined as WBC count of at least $10 \times 10^{9} / \mathrm{L} .{ }^{2}$ Adjusted for age, gender, smoking, body mass index, diabetes, dyslipidemia, and tumor histology (adenocarcinoma vs. non-adenocarcinoma). ${ }^{3}$ Adjusted for age, gender, smoking, body mass index, diabetes, dyslipidemia, and tumor stage (localized stage vs. distant metastasis). Abbreviations: $\mathrm{VTE}=$ venous thromboembolism; $\mathrm{OR}=$ Odds Ratio; $\mathrm{WBC}=$ white blood cell.

in our study had hypertension. The link between hypertension and cancer-associated VTE has received relatively little attention to date. Systemic low-grade inflammation, which was measured based on the level of leukocytes, is involved in the development of atherosclerosis and coronary heart disease, and leukocytosis may be a predictor of cardiovascular events ${ }^{31-33}$. We analyzed the association of leukocytosis and hypertension with VTE. Leukocytosis was linearly associated with hypertension and VTE $(P$ for trend $=0.006)$. The ORs for VTE increased with leukocytosis (all $P$ for trend $<0.05$ ). Hypertension was likely associated with disorders in the system of blood hemostasis, endothelial dysfunction and vascular inflammation, which resulted in an increased risk of thrombosis.

Our study has some limitations. First, although this study was based on only population of lung cancer patients, it was a short-term survey. Second, the larger sample size is needed to provide sufficient power to detect associations of CVD risk factors and VTE risk. Finally, our study lies in the fact that the presence or absence of CVD risk factors for VTE was often not reported in detail.

In conclusion, our study provides data for patients with newly diagnosed lung cancer. Although our results did not establish a causative role of the CVD risk factors in VTE, they suggest the existence of a link between hypertension and cancer-associated VTE. Future prospective studies should further investigate the mechanism underlying this relationship.

\section{Patients and Methods}

Study population. From January 2009 to January 2011, unselected patients with newly diagnosed lung cancer from five hospitals that met the following inclusion criteria were included: histological confirmation of diagnosis; willingness to participate; and provided written informed consent. The exclusion criteria were as follows: any surgery, chemotherapy, or radiotherapy within the past three months; the presence of overt bacteria or a viral infection; a history of VTE (VTE diagnosis at least three months prior to recruitment); and continuous anticoagulation with vitamin $\mathrm{K}$ antagonists or low-molecular-weight heparins. The study was approved by the Central Ethics Committees of Beijing Chao-Yang Hospital of Capital Medical University (No. 2009-4). Data were collected from all of the patients. The methods were carried out in accordance with approved guide-lines.

Measurement of CVD risk factors. CVD risk factors were collected at the baseline examination. The pack-years of smoking were calculated by multiplying the average number of cigarettes per day by the number of years smoked and dividing by 20 . The patients were classified as never, former, or current smokers. The patients were asked to bring all of their current medications, and the medication types, including lipid-lowering 
medications, beta-blockers, angiotensin-converting enzyme inhibitors, and other antihypertensive medications, were recorded. Anthropometrics, including weight and height, were obtained while the patient was wearing a scrub suit. The body mass index was calculated as the weight in kilograms divided by the square of the height in meters $\left(\mathrm{kg} / \mathrm{m}^{2}\right)$. The blood cell counts and lipids and glucose contents were measured before initial treatment. Leukocytosis was defined as a white blood cell count (WBC) of at least $10 \times 10^{9}$ cells/L based on the upper normal limits of a central reference laboratory. Diabetes was defined as a fasting serum glucose level of at least $7.0 \mathrm{mM}$ $(126 \mathrm{mg} / \mathrm{dl})$, a non-fasting glucose level of at least $11.1 \mathrm{mM}(200 \mathrm{mg} / \mathrm{dl})$, or a physician diagnosis of diabetes. Hypertension was defined as a seated diastolic blood pressure of at least $90 \mathrm{mmHg}$, a systolic blood pressure of at least $140 \mathrm{mmHg}$, or a physician diagnosis of hypertension. Dyslipidemia was defined as a total cholesterol level of at least $6.22 \mathrm{mmol} / \mathrm{L}(240 \mathrm{mg} / \mathrm{dl})$, a triglyceride level of at least $2.26 \mathrm{mmol} / \mathrm{L}(200 \mathrm{mg} / \mathrm{dl})$, or a physician diagnosis of dyslipidemia.

Diagnosis and classification of VTE. All of the included patients with newly diagnosed lung cancer were examined for VTE using imaging techniques within one week after admission to the hospital. Furthermore, data for all of patients for the three months prior to recruitment (medical history, presenting symptoms, diagnosis, treatment practices and follow-up data) were retrospectively collected.

DVT events were confirmed by venous ultrasound imaging or a computed tomography venous angiogram. PE events were confirmed by a computed tomography pulmonary angiogram or a ventilation-perfusion scan (if patients had renal insufficiency or allergy to contrast). All VTE events were evaluated by the independent adjudication committee ${ }^{34}$.

Statistical analysis. The continuous variables were summarized as the medians with interquartile ranges or the means and standard deviations (SDs), and the differences between groups were tested with Student's $t$ test for normally distributed variables and with the Wilcoxon rank sum test for non-normally distributed variables. For the categorical variables, the percentages of patients in each category were calculated. The clinical characteristics were compared between subgroups of patients with and without VTE using the Chi-square test or Fisher's exact test, as appropriate. Multiple logistic regression analysis was used to identify factors that were independently associated with VTE in patients with lung cancer. A $P$ value of less than 0.05 was considered statistically significant. All of the analyses were performed using SPSS software for Windows (Version 19.0).

\section{References}

1. Chew, H. K. et al. The incidence of venous thromboembolism among patients with primary lung cancer. J Thromb Haemost 6 , 601-608 (2008).

2. Corrales-Rodriguez, L. \& Blais, N. Lung cancer associated venous thromboembolic disease: a comprehensive review. Lung Cancer 75, 1-8 (2012)

3. Connolly, G. C., Dalal, M., Lin, J. \& Khorana, A. A. Incidence and predictors of venous thromboembolism (VTE) among ambulatory patients with lung cancer. Lung Cancer 78, 253-258 (2012).

4. Ogren, M., Bergqvist, D., Wahlander, K., Eriksson, H. \& Sternby, N. H. Trousseau's syndrome - what is the evidence ? A populationbased autopsy study. Thromb Haemost 95, 541-545 (2006).

5. Prandoni, P., Falanga, A. \& Piccioli, A. Cancer and venous thromboembolism. Lancet Oncol 6, 401-410 (2005).

6. Connolly, G. C. \& Francis, C. W. Cancer-associated thrombosis. Hematology Am Soc Hematol Educ Program 2013, 684-691 (2013).

7. Blom, J. W., Osanto, S. \& Rosendaal, F. R. The risk of a venous thrombotic event in lung cancer patients: higher risk for adenocarcinoma than squamous cell carcinoma. J Thromb Haemost 2, 1760-1765 (2004).

8. Ageno, W., Becattini, C., Brighton, T., Selby, R. \& Kamphuisen, P. W. Cardiovascular risk factors and venous thromboembolism: a meta-analysis. Circulation 117, 93-102 (2008).

9. Piazza, G. Venous thromboembolism and cancer. Circulation 128, 2614-2618 (2013).

10. Calip, G. S. et al. Metabolic syndrome and outcomes following early-stage breast cancer. Breast Cancer Res Treat 2014, 10 (2014).

11. Okazaki, I., Ishikawa, S. \& Sohara, Y. Genes Associated with Succeptibility to Lung Adenocarcinoma Among Never Smokers Suggest the Mechanism of Disease. Anticancer Res 34, 5229-5240 (2014).

12. Yin, X. H., Jia, H. Y., Xue, X. R., Yang, S. Z. \& Wang, Z. Q. Clinical analysis of endometrial cancer patients with obesity, diabetes, and hypertension. Int J Clin Exp Med 7, 736-743 (2014).

13. Friedenreich, C. M. et al. Case-control study of the metabolic syndrome and metabolic risk factors for endometrial cancer. Cancer Epidemiol Biomarkers Prev 20, 2384-2395 (2011).

14. Prandoni, P. et al. An association between atherosclerosis and venous thrombosis. N Engl J Med 348, 1435-1441 (2003).

15. Hong, C. et al. Coronary artery calcification and risk factors for atherosclerosis in patients with venous thromboembolism. Atherosclerosis 183, 169-174 (2005).

16. van der Hagen, P. B. et al. Subclinical atherosclerosis and the risk of future venous thrombosis in the Cardiovascular Health Study. $J$ Thromb Haemost 4, 1903-1908 (2006).

17. Reich, L. M. et al. Prospective study of subclinical atherosclerosis as a risk factor for venous thromboembolism. J Thromb Haemost 4, 1909-1913 (2006).

18. Tsai, A. W. et al. Cardiovascular risk factors and venous thromboembolism incidence: the longitudinal investigation of thromboembolism etiology. Arch Intern Med 162, 1182-1189 (2002).

19. Holst, A. G., Jensen, G. \& Prescott, E. Risk factors for venous thromboembolism: results from the Copenhagen City Heart Study. Circulation 121, 1896-1903 (2010).

20. Lutsey, P. L. et al. Correlates and consequences of venous thromboembolism: The Iowa Women's Health Study. Am J Public Health 100, 1506-1513 (2010).

21. Wattanakit, K. et al. Association between cardiovascular disease risk factors and occurrence of venous thromboembolism. A timedependent analysis. Thromb Haemost 108, 508-515 (2012).

22. Goldhaber, S. Z. et al. A prospective study of risk factors for pulmonary embolism in women. Jama 277, 642-645 (1997).

23. Severinsen, M. T. et al. Smoking and venous thromboembolism: a Danish follow-up study. J Thromb Haemost 7, 1297-1303 (2009).

24. Hansson, P. O., Eriksson, H., Welin, L., Svardsudd, K. \& Wilhelmsen, L. Smoking and abdominal obesity: risk factors for venous thromboembolism among middle-aged men: "the study of men born in 1913". Arch Intern Med 159, 1886-1890 (1999).

25. Pomp, E. R., Rosendaal, F. R. \& Doggen, C. J. Smoking increases the risk of venous thrombosis and acts synergistically with oral contraceptive use. Am J Hematol 83, 97-102 (2008). 
26. Lee, K. W. \& Lip, G. Y. Effects of lifestyle on hemostasis, fibrinolysis, and platelet reactivity: a systematic review. Arch Intern Med 163, 2368-2392 (2003).

27. Mahmoodi, B. K. et al. Microalbuminuria and risk of venous thromboembolism. Jama 301, 1790-1797 (2009).

28. Heit, J. A. et al. Is diabetes mellitus an independent risk factor for venous thromboembolism? a population-based case-control study. Arterioscler Thromb Vasc Biol 29, 1399-1405 (2009).

29. Chamberlain, A. M., Folsom, A. R., Heckbert, S. R., Rosamond, W. D. \& Cushman, M. High-density lipoprotein cholesterol and venous thromboembolism in the Longitudinal Investigation of Thromboembolism Etiology (LITE). Blood 112, 2675-2680 (2008).

30. Deguchi, H., Pecheniuk, N. M., Elias, D. J., Averell, P. M. \& Griffin, J. H. High-density lipoprotein deficiency and dyslipoproteinemia associated with venous thrombosis in men. Circulation 112, 893-899 (2005).

31. Yarnell, J. W., Patterson, C. C., Sweetnam, P. M. \& Lowe, G. D. Haemostatic/inflammatory markers predict 10-year risk of IHD at least as well as lipids: the Caerphilly collaborative studies. Eur Heart J 25, 1049-1056 (2004).

32. Soderholm, M., Zia, E., Hedblad, B. \& Engstrom, G. Leukocyte count and incidence of subarachnoid haemorrhage: a prospective cohort study. BMC Neurol 14, 71 (2014).

33. Kalburgi, V. et al. Role of systemic markers in periodontal diseases: a possible inflammatory burden and risk factor for cardiovascular diseases? Ann Med Health Sci Res 4, 388-392 (2014).

34. Zhang, Y. et al. Prevalence and associations of venous thromboembolism in patients with newly diagnosed lung cancer. Chest 2014, 13-2379 (2014).

\section{Acknowledgements}

This study was supported in part by grants from the China Key Research Projects of the 12th National FiveYear Development Plan (Grant No. 2012BAI05B02), National Natural Science Foundation of China (Grant No. 31570890), and in part by the Wu Jieping Medical Foundation (Grant No. 320.6750.14286). The authors would like to thank the members of the independent adjudication committee (Lei Zhang, Zhanhong Ma, and Liming Zhang) for evaluation of VTE in this study.

\section{Author Contributions}

C.W. and Y.H.Z. conceived and designed the study. Y.H.Z., W.H.C., Y.H.Y., L.J.G. and Z.G.Z., and the China VTE Study Group (L.Z. of Peking Union Medical College Hospital, Chinese Academy of Medical Sciences; L.N.J. of The First Affiliated Hospital of Bengbu Medical College; Q.X.X. of The Affiliated Hospital of Jining Medical College; and X.H.Z. of The Navy General Hospital of PLA) performed the study. L.R.L. and Y.H.Z. analyzed the data. Y.H.Z. and C.W. contributed to the writing of the manuscript. All the authors reviewed and approved the final version of the manuscript.

\section{Additional Information}

Competing financial interests: The authors declare no competing financial interests.

How to cite this article: Zhang, Y. et al. Hypertension associated with venous thromboembolism in patients with newly diagnosed lung cancer. Sci. Rep. 6, 19603; doi: 10.1038/srep19603 (2016).

(c) (i) This work is licensed under a Creative Commons Attribution 4.0 International License. The images or other third party material in this article are included in the article's Creative Commons license, unless indicated otherwise in the credit line; if the material is not included under the Creative Commons license, users will need to obtain permission from the license holder to reproduce the material. To view a copy of this license, visit http://creativecommons.org/licenses/by/4.0/

\section{Consortia}

Li Zhang ${ }^{3}$, Qixia Xu' ${ }^{4}$ Luning Jiang ${ }^{5}$, Xinhong Zhang ${ }^{6}$

${ }^{3}$ Department of Respiratory Medicine, Peking Union Medical College Hospital, Chinese Academy of Medical Sciences, Beijing, 100730, China; ${ }^{4}$ Department of Respiratory and Critical Care Medicine, the First Affiliated Hospital of Bengbu Medical College, Bengbu, 233004, China; ${ }^{5}$ Department of Respiratory Medicine, The Affiliated Hospital of Jining Medical College, Jining, 272001, China; ${ }^{6}$ Department of Oncology, The Navy General Hospital of PLA, Beijing, 100048, China. 